http://jmscr.igmpublication.org/home/ ISSN (e)-2347-176x ISSN (p) 2455-0450 crossref DOI: https://dx.doi.org/10.18535/jmscr/v8i8.57

\title{
Management of a Symptomatic Giant Hemangioma of Liver- A Case Report Study
}

\author{
Authors \\ Karan Singh Thakur ${ }^{1}$, Purnima Patial ${ }^{2 *}$ \\ ${ }^{1}$ Resident Doctor, Dept. of Surgery PGI Chandigarh, India \\ ${ }^{2}$ Resident Doctor, Dept. of Medicine, RPGMC Tanda, HP, India \\ *Corresponding Author \\ Purnima Patial
}

\begin{abstract}
Hemangiomas are the most common benign tumors of the liver. They are more common in females. There incidence is $0.4 \%-20 \%$. More commonly hemangiomas involve right lobe of liver. Most commonly they are incidental findings. There is no evidence of malignant transformation. Small and medium types require no treatment. Size of liver hemangioma is not a formal indication for surgery. Giant hemangioma with symptoms require treatment. Abdominal pain or discomfort are the most common indication for surgery. Enucleation is the preferred surgical procedure. Recurrence after surgery is uncommon. Surgery for hemangioma of liver is less frequently being performed procedure. Thus such a case is discussed in this case report study.

Keywords: Giant hemangioma, Enucleation, Resection.
\end{abstract}

\section{Introduction}

Hemangioma of the liver is the most common liver tumor. This is $70 \%$ of all the benign lesions of the liver. They are clusters of blood filled cavities, lined by endothelial cells. Most often they are discovered incidentally. Typically they are called capillary hemangiomas. Large one are called cavernous hemangiomas. Their average size ranges from $3 \mathrm{~cm}$ to $10 \mathrm{~cm}$. Majority of them are less than $5 \mathrm{~cm}$. Prevalent age is $30-50$ years. Its Prevalence is $3-20 \%$ in the general population. They are more common in women, with a 5:1 female/male ratio. It can occur in all age groups, but mean age of presentation is 45 years. They affect both lobes. Hemangiomas larger than $5 \mathrm{~cm}$ are called "Giant hemagiomas" Surgery is required in symptomatic cases only.

\section{Pathogenesis}

The exact pathology how hemangioma is formed is unknown. Possible genetic connection may be there. Hormones (estrogen/ocp's) may be pathogenic. Mechanism of formation is thought to be vascular malformations orhamartomas of congenitalorigin that enlarge by ectasia rather than by hyperplasia or hypertrophy.

Macroscopically they are well-delineated, reddish blue, flat lesions that maypartially collapse during sectioning. Some amount of fibrosis, calcification and thrombosismay be seen similar to radiological findings.

Microscopically hemangiomas are made of cavernous vascular spaces which are lined by flattened endothelium underlying fibrous septa of various widths. 
Small hemangiomas may become entirely fibrous appearing as "solitary fibrous nodule." There is no evidence of malignant transformation.

Predisposing factors are-

- Adulthood

- Steroid use

- Female sex

- Pregnancy

\section{Clinical Features}

Majority of the liver hemangiomas are asymptomatic, found incidental on ultrasound or during laparotomy for unrelated reasons. Majority of them remain stable in size. However larger lesions show significant higher growthover time. Tumour enlargement of more than 5\% may be observed in upto $45 \%$ of the lesions. Large hemangiomas may be asymtomatic or symptomatic. Symptomatic cases may present with abdominal pain and right upper quadrant discomfort or fullness, decreased appetite, early satiety, nausea, vomiting and rarely as an abdominal mass. Clinical examination is usually normal, however hepatomegaly, palpable mass may be found in few cases.

\section{Complications}

Complications depends on size, are mostly seen in large hemangiomas. Alterations of internal architecture of the hemangioma, such as with inflammation may occur. Inflammation may cause fever. Traumatic or spontaneous mechanical rupture can occur. Coagulation abnormalities can be seen, which could lead to systemic disorders like hemorrhage, rupture and subsequently hemoperitoneum. Large hemangioma can cause compression of adjacent structures like stomach and bile duct. Other complications include Kasabach_ Merrit Syndrome which is a consumptive coagulopathy in children, and causes thrombocytopenia. Rarely torsion and infarction, bleeding may be seen.

\section{Diagnosis}

Most of the hemangiomas are incidental findings.
They are diagnosed during radiology or laparotomy for other cause. Blood investigations are not very helpful in diagnosis.

Thrombocytopenia due to splenic sequestration and destruction may be present. Liver function tests are usually normal, unless complications like thrombosis, bleeding or compression of bliliary tree occur. Alpha-fetoprotein, CEA and CA19-9 are in normal limits.

Mostly diagnosis is made on radiological tests. CT and MRI are usually sufficient to make the diagnosis. Ultrasound is the first approach. USG has sensitivity $94.1 \%$ and specificity $80 \%$. On Ultrasound they appear as homogenous hyperechoic mass with sharp margins less than $3 \mathrm{cms}$. The diagnosis can be strongly suggested by ultrasound in approximately 80 percent of patients with lesions less than $6 \mathrm{~cm}$.

When hemangioma is found on USG, follow up of the patient is done as-

1) Patients with history of known liver disease or suspected extra-hepatic malignancy should undergo a confirmatory examination such as a CE-CT or MRI.

2) In patients with no evidence of liver disease or extra hepatic malignancy and "typical" appearances of hemangioma on USG, an acceptable alternative is to repeat USG at 36 months to document stability.

CT criteria for diagnosis includes-

1) Low attenuation on non-contrast CT.

2) Peripheral and globular enhancement of the lesion, followed by central enhancement on CECT.

3) Contrast enhancement of lesion on delayed phases.

MRI is the key imaging modality in characterization. Hypointense lesion on T1 weighted sequence and strongly enhancing lesion on T2 weighted images are seen in hemangioma.

\section{Treatment}

Most of hepatic hemangioma are smaller in size and are asymptomatic. No treatment is required if hemangioma is asymptomatic. Observation and 
reassurance is usually enough. Follow up with 6 month or annual imaging is done.

If size of the lesion is smaller than $1.5 \mathrm{~cm}$-patient can be simply reassured and observed.

If size is $5 \mathrm{~cm}$ or smaller-follow-up imaging is not required provided there is certainty of the diagnosis based on radiologic and on clinical details.

In lesions larger than $5 \mathrm{~cm}$ - Repeat imaging is done in 6 to 12 months, using the imaging modality that best showed the hemangioma previously (eg, CT or MRI).

If there is no change in the size of the lesion, there is no need to perform additional imaging.

Size only is not an indication for surgery.

Indications of treatment include-

- Severe symptoms

- Complications

- Fever of unknown origin with a liver mass

- Diagnostic uncertainity- inability to exclude malignancy

- Growing lesion on serial imagingMinimum 25\% increase in diameter over 6 months

- And anxiety of the patient.

Prophylactic surgical intervention in liver hemangioma may be considered in- Giant hemangioma more than $10 \mathrm{~cm}$ with pain and hemangioma adjacent to major vascular structure.

Surgical resection/enucleation remains definitive treatment. However, prior to surgery, it is important to evaluate and exclude other causes of pain. Enucleation is preferred in hemangiomas located inperipheral liver areas. Resection is required for deeply located lesion, multiple hemangiomas and lesions adjacent to vascular structures. Segmental resections or enucleation can be performed by open or laparoscopic approach. Morbidity is seen in $10 \%-27 \%$ cases while mortality may be there in $2 \%$ cases. Recurrence is seen in upto $4 \%$ cases. Right or left hepatectomy indicated for large mass occupying entire lobe.

Other less effective portion of treatment includes: hepatic artery ligation, embolization of feeding vessel and radiation therapy. RFA may be considered as an alternative treatment for size 5$10 \mathrm{~cm}$. There are only few cases in literature of liver transplant for this disease. Liver transplantation can be considered in exceptional situations to treat technically unresectable complicated giant hemangiomas with cardiopulmonary complications and in large diffuse bilateral lesion.Role of other modalities under study are- sorafenib, propranolol and bevacizumab.

\section{Case Study}

A 46 years old female patient presented with complaints of pain in her Upper Abdomen for 2 years. Patient had recurrent intermittent episodes of mild to moderate intensity pain. She had no relief in symptoms with medications. She had vomiting episodes for 2 months. She also had feeling of abdominal fullness and discomfort. There were no complaints like fever, jaundice, black colored stool, loss of weight, loss of appetite and upper or lower GI bleed. She never hadsuch complaints in past. She didn't have anycomorbidities. She was vegetarian, non-smoker and non-alcoholic.

Her general physical examination and vitals were normal. On her abdomen examination she had mild tenderness in right hypochondrium. Otherwise her abdomen was soft, non-distended. No plapable lump was noticed. Per rectal examination was normal.

Blood investiagations including LFT's, coagulogram, tumor markers AFP, CEA and CA19-9 were normal.USG Abdomen showed ill defined heterogenous hypoechoic lesion in the liver. CECT Abdomen showed a large exophytic lobulated hypodense lesion approximately 9.1X9.7X5.9 $\mathrm{cm}$ in Segment 5 and 6 with multipleareas of low density areas within it.On post contrast images it shows discontinuous peripheral nodular enhancement and progressive centripetal filling on delayed phase with few non enhancing areas s/o necrosis. Findings were suggestive of giant hemangioma liver. 


\section{JMSCR Vol||08||Issue||08||Page 341-345||August}

So based on the above investigations diagnosis of symptomatic Giant liver hemangioma was made. Hence patient was planned for surgical resection of the mass.

Patient was operated under general anaesthesia. Intraoperatively there was no ascitis. No liver, omental, peritoneal, or mesentric deposits were found. A firm mass of size $10 \times 8 \times 7 \mathrm{~cm}$ arising from liver segment 5 and 6 was noticed, reaching upto duodenum, hepatic flexure and hepatoduodenal ligament. Ligation of feeding vessel done. Enucleation of the hemangioma was done.

Histopathological examination report of the removed specimen came out to be cavernous hemangioma.

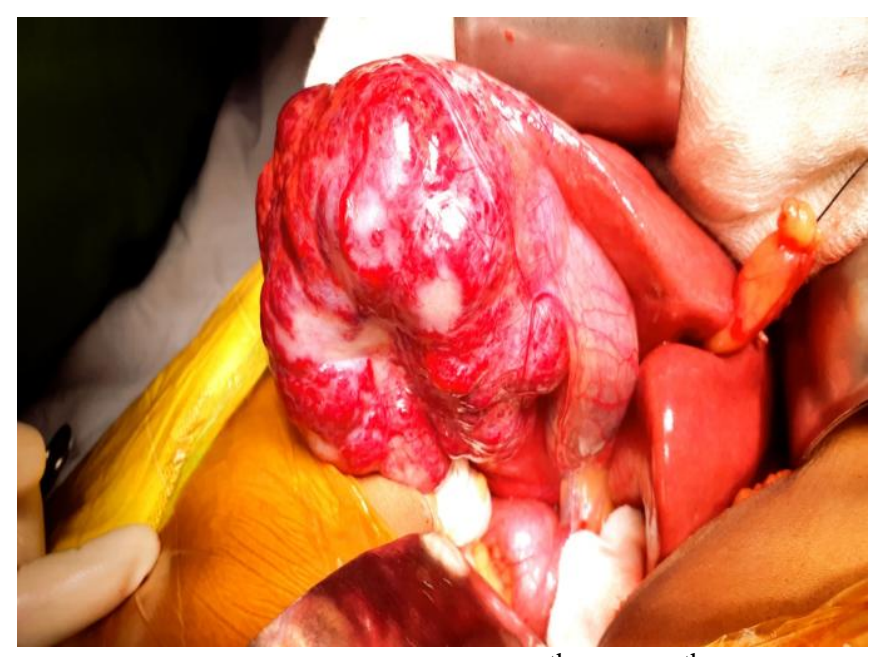

Fig.1- Giant Hemangioma in $5^{\text {th }}$ and $6^{\text {th }}$ segment of liver

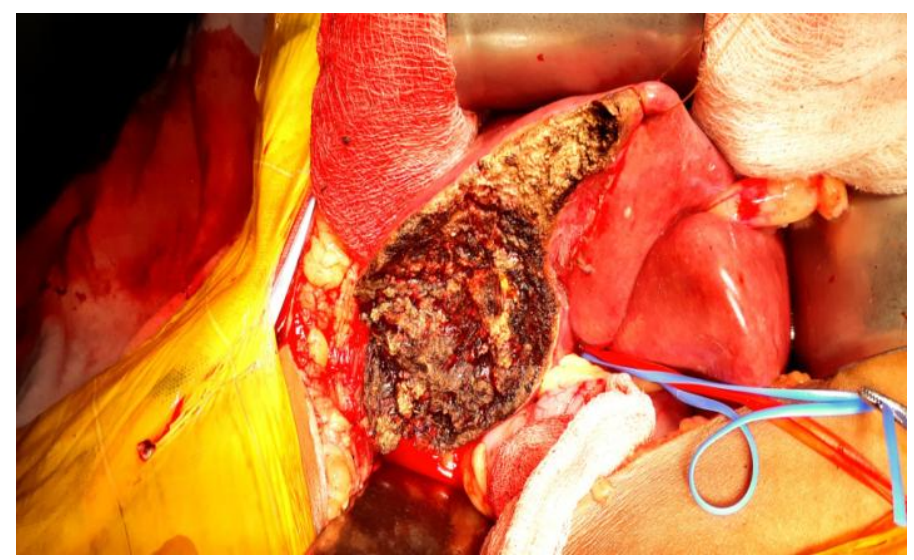

Fig. 2- Liver surface after enucleation of the hemangioma

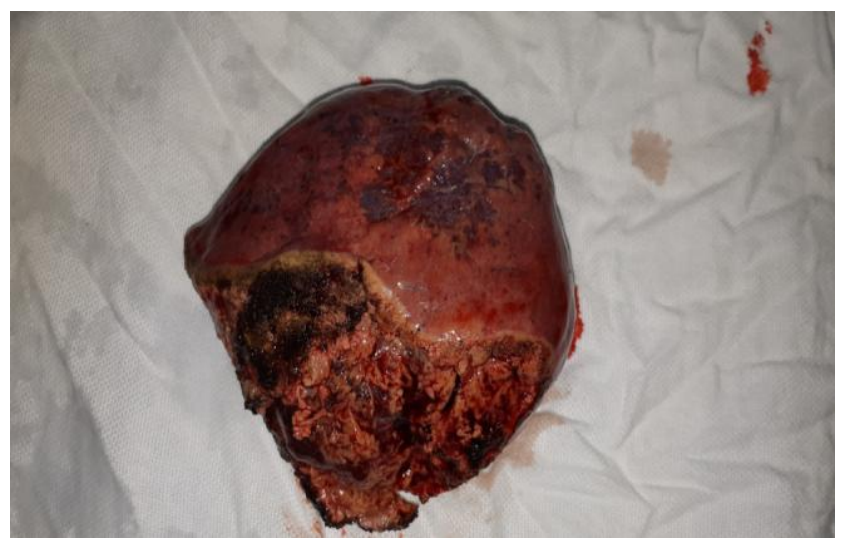

Fig. 3- Removed out specimen of hemangioma

Post operative course in the hospital was uneventful. She was discharged in a complete hemodynamically stable condition on postoperative day 6. Regular follow-ups were done. During her follow-ups patient was completely symptoms free. No recurrence was found in the subsequent visits.

\section{Observation and Result}

This is a case report of a symptomatic hemangioma patient. Hepatic hemangiomas are mostly asymptomatic. Size is not a criterion for surgical intervention. Here in this case hemangioma was not an incidental finding, rather she was diagnosed after she developed symptoms. Her symptoms were due to compressive effects of the lesion. She was not responding to conservative approach as the size of the hemangioma was big. So conservative approach was not much helpful to the patient.USG and CT helped in confirming the diagnosis. After confirmation of the diagnosis surgical intervention was planned. Enucleation of the Giant hepatic hemangioma was performed. Outcome after surgery was good. No complications occurred. No recurrence was seen during regular follow-ups.

\section{Discussion}

Hepatic hemangiomas are mainly incidental findings. Most of them are asymptomatic. They have no malignant potential. So in most of the cases only observation and reassurance is required. However, hemangiomas may be symptomatic in few cases. Size is not an 
indication to intervene. Intervention is required, when the hemangiomas become symptomatic. Mostly symptoms are due to compression of the adjacent structures. Management is not much complex. Ultrasound is the initial investigation of choice. Further confirmation and characterization can be done with CT or MRI. Surgical resection by enucleation is the preferred procedure. Outcome is usually good. Recurrence is uncommon.

Most surgeons encounter hemangiomas incidentally during laparotomy for other diseases. However surgery for hemangiomas is very less commonly performed in the operating rooms. Thus disease course and the management plan should be clear to the clinician attending such case.

\section{References}

1. Jarnagin WR, Allen PJ, Vilgrain V, Cauchy F. Benign liver lesions, $6^{\text {th }}$ ed. Blumgart'sSurgery of the Liver, Biliary Tract, and Pancreas: 1299-1317.

2. Norman SW, Christopher JK, Ronan P. The Liver, $27^{\text {th }}$ ed. Bailey and Love Short Practice of Surgery: 1153-73.

3. Courtney MT, Daniel B, Mark, E, Kenneth LM. The Liver, $20^{\text {th }}$ ed. Sabiston textbook of Surgery: 1455-58.

4. Belghiti J, Cauchy F, Paradis V, et al. Diagnosis and management of solid benign liver lesions. Nat Rev Gastroenterol Hepatol. 2014;11:737-49.

5. Yedibela S, Alibek S, Müller V, et al. Management of hemangioma of the liver: Surgical therapy or observation? World J Surg. 2013;37:1303.

6. Zinner MJ, Ashley SW, Cho Cs, Fong Y. Benign and Malignant primary Liver neoplasms, $12^{\text {th }} \mathrm{ed}$. Maingot's Abdominal Operations: 927-48. 